\title{
Modeling Social-Ecological Problems in Coastal Ecosystems: A Case Study
}

\author{
JOHN FORRESTER, ${ }^{1}$ RICHARD GREAVES, ${ }^{2}$ HOWARD NOBLE, ${ }^{3}$ AND RICHARD TAYLOR ${ }^{4}$ \\ ${ }^{1}$ York Centre for Complex Systems Analysis, Ron Cooke Hub, University of York, York, YO10 5GE, United \\ Kingdom; ${ }^{2}$ Department of Computer Science, University of York, York, YO10 5GE, United Kingdom; \\ ${ }^{3}$ Oxford University IT Services, 13 Banbury Road, Oxford, OX2 6NN, United Kingdom; and ${ }^{4}$ Stockholm \\ Environment Institute, Florence House, 29 Grove Street, Oxford, OX2 7JT, United Kingdom
}

Received 7 January 2013; accepted 3 March 2014

\begin{abstract}
Complex social-ecological systems (SES) are not amenable to simple mathematical modeling. However, to address critical issues in SES (e.g., understanding ecological resiliencelamelioration of poverty) it is necessary to describe such systems in their entirety. Based on empirical knowledge of local stakeholders and experts, we mapped their conceptions of one SES. Modelers codified what actors told us into two models: a local-level model and an overarching multiple-entity description of the system. Looking at these two representations together helps us understand links between the locally specific and other levels of decision taking and vice-versa. This "bimodeling" approach is investigated in one SES in coastal Kenya. (C) 2014 Wiley Periodicals, Inc. Complexity 19: 73-82, 2014
\end{abstract}

Key Words: agent-based models; unified modeling language; participatory modeling

\section{INTRODUCTION}

gent-based models (ABMs) often used to simulate aspects of the natural environment can appear incredibly accurate; yet using ABMs to support important policy decisions amongst people appears to

(C) 2014 The Authors. Complexity Published by Wiley Periodicals, Inc. This is an open access article under the terms of the Creative Commons Attribution License, which permits use, distribution and reproduction in any medium, provided the original work is properly cited.

Correspondence to: John Forrester, York Centre for Complex Systems Analysis, Ron Cooke Hub, University of York, York, YO10 5GE, United Kingdom.

E-mail: john.forrester@york.ac.uk face multiple obstacles in terms of the modeler's ability to generate a similar level of accurate representation. One key reason for this apparent performance differential is that natural science modelers model a minimum number of aspects, while social modelers typically include many factors. Thus, while ecological systems are complex, social systems are complex and "messy" $[1,2]$. Modeling socialecological systems (SES) involves attempting to represent complexity and this "messiness."

This problem can also be found encapsulated in the KISS (keep it simple, stupid) or KIDS (keep it descriptive) debate [3]. While those modeling "simple" complex systems (i.e., ones which exist largely within one disciplinary domain) often argue for KISS, the KIDS approach has a major part to play in making better informed, more policy-relevant models of human action; the descriptive 
representation of aspects is important. There may also be a fundamental difference in what each is trying to do: in general, it is defensible to say that "simple" complex systems modelers are trying to simulate (i.e., mimic) the behavior of their subjects so as to understand its patterns; while modeling done to inform policy has a more complicated purpose, which involves and includes concerns of the modelers and those of the end users, often the policy makers, policy advisors, or other decision takers. That said, the latter is also to do with the way the models themselves (or scenarios produced with them) are used: this article is primarily concerned with how the modeling itself is conceived to best represent SES.

The article is a contextualized discussion of what we did to model one particular SES. Coastal Kenya supports the livelihoods of millions, putting vital natural resources under increasing strain. Artisanal fishery supports a large and varied set of livelihoods; thus, fisheries provide an "ecosystem service." Fishing grounds, including public beach and fish landing sites, are under the authority of Beach Management Units (BMU), which are made up of representatives from the local fishing and fish and shell trading communities. While the environmental impact of fishing is widely acknowledged, there are differing views among the BMUs and other actors as to what measures are appropriate to sustain the fishery and protect reefs. Solutions lie in understanding how and why people's actions contribute to impact. We needed a structured robust way of cocreating and communicating data about actions, causes, and outcomes across geographic distance and across levels of decision taking (i.e., up to higher level policy makers and from scientific advisors to policy makers and end users). Our case study (partly funded by the ESPA programme: see http://www.espa.ac.uk/) was to develop conceptual representative tools and models built on existing knowledge in the region, working with local teams, and connecting with policy makers, practitioners, and the poorer people who depend directly on the ecosystem service.

This article, thus, describes our work on modeling the link between human action and an ecosystem service, in a project that looked into the wider aspects of (social) knowledge networks and (institutional) decision-making structures. We identify modeling challenges in relation to dealing with different epistemological backgrounds, the integration of different stakeholder worldviews and knowledge(s), and other bottlenecks in the formalization process. The process is described and discussed in relation to the example case from which initial ideas and understandings were derived.

Our project used the philosophy of "structured subjectivity" [4] to help describe aspects of SES networks regarding decision taking surrounding coastal production systems in Kenya. Structured subjective methods are designed to provide formal outputs that we found can be more readily included in models but still reflect actors' conceptions of the system. Outputs of such approaches can include statistically valid understanding of actor beliefs (using Q-methodology), network maps of actors (using social network mapping), codification of their "tacit" behavioral rules and decision logic (e.g., using tools for knowledge elicitation [5]), and cocreation of formal "cartoon" models (e.g., using unified modeling language [UML]).

The empirical approach we will discuss is twofold: using rigorous "bottom-up" (participatory) methods to inform an $\mathrm{ABM}$ but doing this within an overarching framework provided by the highly structured UML "cartoon" model. This overarching construction allows a locally specific KIDS approach to be applied in the detailed modeling and simulation stages, while allowing the conceptual models to remain more generally described. Thus, while it probably remains impossible to model the full range of human behavior, we could successfully simulation model the behaviors of fishers that change the environment (i.e., their boat and gear). This can then be understood, and communicated, on a wider scale within the series of UML diagrams.

\section{BACKGROUND, INCLUDING THE SETTING OF THE PROBLEM}

For more than 10 years, colleagues have been identifying "a need for a systems approach" which "can identify interlinkages and flow of information and decision making processes" [6] with respect to SES. In natural sciences, as in economics, it is common to use models inspired by systems thinking and it is commonplace to, by virtue of their formality, attribute these models more scientific merit than their descriptive counterparts that are usually used to represent social elements. Social studies which would look at information and decision processes are usually carried out without the use of formalized or mathematical models, where researchers plump instead for a social conceptualisation or else by the use of quasi-economic proxies [7]. The "problem" with these approaches is that, as Coulthard et al. also point out, "the quality of life or subjective wellbeing element of the framework is only one dimension of what needs to be understood."

The process of modeling social elements has been described very simply by deVries and Petersen as first "analyze people's value orientations and the way in which they interpret sustainability problems i.e. their beliefs"; then "translate the resulting worldviews into model-based narratives, i.e. scenarios"; and then "investigate outputs in terms of associated risks and opportunities and robustness of policy options" [8]. In terms of formal modeling then, the translation step is one in which actors' worldviews are codified and, in doing so, many assumptions are made explicit. This presents an opportunity to clarify stakeholders' perceptions within a coconstruction process with the 
stakeholders themselves. Scenario building offers another point of integration of modeling effort with stakeholder priorities. Recent research has also shown the value of the design of "what-if" scenarios in collaboration with resource managers, when used in the exploration of more context-specific models [9].

Nonetheless, scenario-building introduces a potential problem; the question of whether the assumptions informing scenarios may be shared or not. From a modeling point of view, a shared understanding may be preferred because it has the advantage of simplifying a model, thus, reducing the number of needed model variations. However, it is not clear whether any apparent consensual agreement is valid. Most importantly, to represent social "messiness" we need to avoid dominant worldviews controlling outputs when there are other equally relevant insights. Agent-based modeling permits significant diversity through disaggregation of populations into actor subtypes and attributes of individuals. Conversely, many of the model assumptions are also global. One approach is the use of modular construction, which makes it feasible that certain aspects of the model could be activated or deactivated, or substituted, to account for differing interpretations. Seen in this light, there is also the need to avoid that idea that researchers are presenting a given simulation model as a "solution" to understand an issue: the participatory nature of the coconstruction of ABMs and scenario-building activities encourages us to introduce several different shades of any simulation model to show different possibilities.

The companion modeling approach (ComMod-see also http://www.commod.org) has, in the past 15 years, developed a range of activities to support the use of models in natural resource management and in addressing social-ecological interactions in particular $[10,11]$. Others have developed the methodology for the coconstruction of conceptual models [12,13]. Such models visually represent multiple viewpoints and can be used as mediating and discursive objects that promote collective learning processes. Further, they can be used to formulate ABMs. Their design depends on the research question identified-and if it is stakeholder-driven research it often depends on the "overarching negotiated development question" [14]. This "ComMod" approach provides one solution to our "shared understanding" issue.

Notwithstanding, any model that looks only at the behavior of actors in one sector (be it economic, political, or resource-use spheres) will miss critical dynamic processes in other sectors. Kemp-Benedict et al. note that "The range of possible behaviors is not infinite-human agency is bounded by physical constraints, social norms, family behaviors, and physiological endowments. However, the range can be large and the interactions of these elements, complex" [15]. Thus, while the task is not impossible it is difficult and time consuming. However, in our ESPA pro- ject (see http://www.espa.ac.uk/projects/ne-i00288x-1 and http://weadapt.org/search?q=WD-NACE), we were not only trying to link "sectors" and introduce a dynamic ecological aspect into social models but also link across levels of decision making. Thus, we needed an overarching approach which would allow us not only to link sectors but also "scale up."

Taking our inspiration from the complex systems modeling and simulation (CoSMoS) $[16,17]$ experience in successfully linking complex systems modelers and software engineers with research scientists in biomedicine and robotics, we separated out the processes of representing the whole system (the "domain") from the simulation process. For the simulation, we used ABMs; and we used UML [1820] to represent the "domain" or "conceptual" model [21]. However, we did not use the "full" CoSMoS approach as outlined in Figure 1 as our simulation models (ABMs) were created using the Modelling4All (www.modelling4all.org/ about/) approach as described later and not derived directly from the UML process, as might be expected. The implication of this bilevel, bimodeling approach was that we needed to work on transdisciplinary understandings of the whole system: in particular, we needed conceptual modeler/simulation modeler counderstanding of the approach, and modeler/social science counderstanding.

The CoSMoS approach encourages us not to move directly from the "domain" (i.e., real world) to simulation but to create a "domain model" first, that is, agreed on between the modeler and the domain experts. Figure 1 shows all the stages of the CoSMoS approach and how they are linked. Superimposed on the original diagram are three ellipses showing where in particular a transdisciplinary understanding is required. The domain may remain largely the preserve of those who know about the reality of ecosystem services and society. The simulation modeling can be chiefly the preserve of the computer science modelers. In the middle (dotted ellipse), however, is an area of explicit transdisciplinarity where domain expert(s) and programmer must engage. This includes the creation of the domain model and the analysis of simulations.

Modelers and domain experts are readily able to use the above approach, where the domain model follows the KISS principle. In fact, there are practical reasons as well as scientific reasons for doing so such as to improve the model's generality as well as tractability. However, other colleagues [3] argue for a "messy" approach even in the domain model, as the importance of understanding and representing the social correctly remains important because people's cognitive maps guide them in transforming their norms and values into actions. However, our aim was a structured KIDS approach, recognizing that KISS can be one important type of entry point and interface for engagement between modelers and domain experts but trying to retain social detail. 


\section{FIGURE 1}

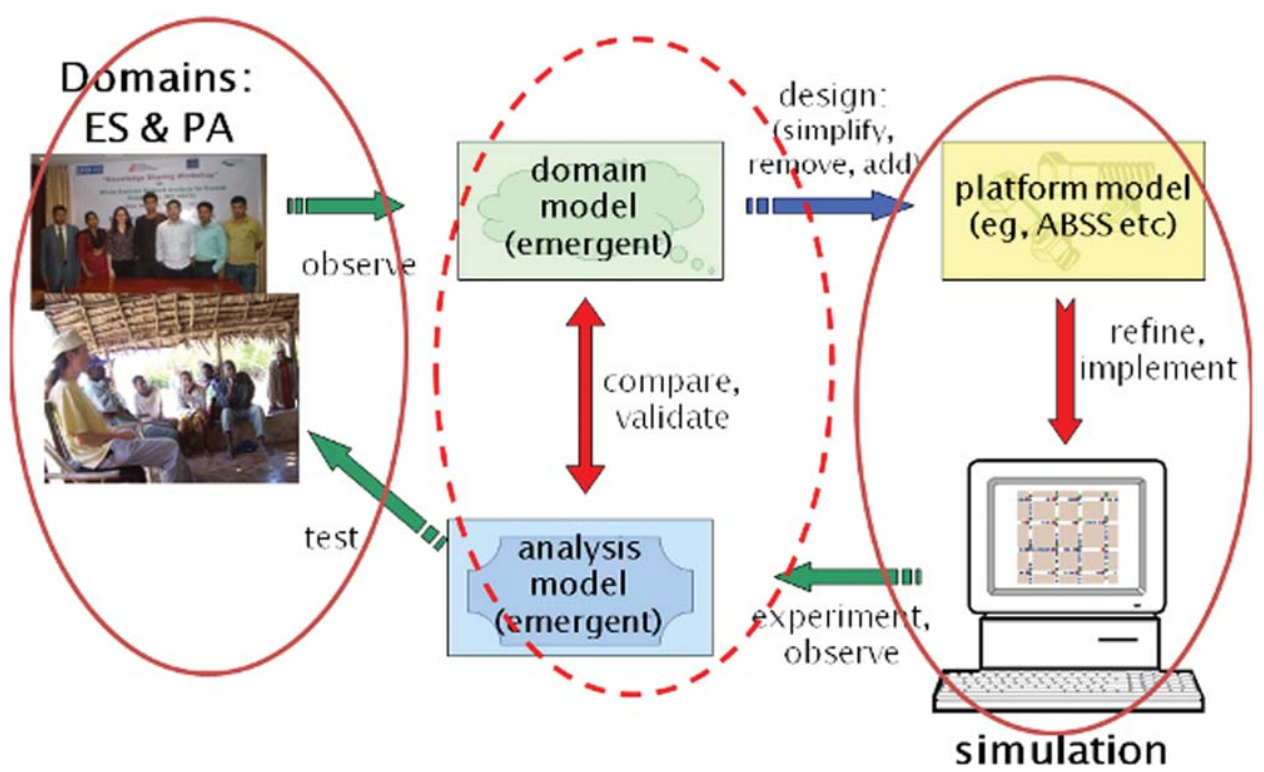

How the CoSMoS approach should be implemented in theory-with thanks to Prof. Susan Stepney, York Centre for Complex Systems Analysis (YCCSA), University of York.

When we used the CoSMoS approach it had not yet been tested with cases embodying a structured KIDS approach: our experience was that, at the level of the direct resource users, the conceptual model (CoSMoS "domain model") became quite complicated, and it became a difficult documentation task to describe the links and interactions in any detail in the UML, although the level of ABM simulation worked well. However, producing a broad-brush-stroke, overarching model of the combined SES was at least possible using UML.

But within the overarching framework provided by the UML, researchers, end users, and policy decision takers could start to understand the relationship between the ABMs of social/ecological consequences and other models of the ecological system (e.g., using equational modeling packages). This article does not deal with the ecological models explicitly, but we have reason to believe that linking ecological and relatively complex social issues at one spatial scale has already been done [22] and is, thus, considered less problematic. What remains to be described is the issue of creating and then understanding how to link, layer, or nest the discrete simulation models of the bits of the system within the conceptual overarching model of the whole system.

\section{ON THE EXPERIENCE OF "BOTTOM-UP" MODELING (USING ABMS)}

We were unequivocal from the start that our ABMs would be informed by the knowledge held by local actors.
Thus, at the local, social level, we looked at what people think, what they know (and what they think they know), rather than just "hard" facts. However, we had to recognize that there is a level of complexity in the thought processes of real actors: the "explicit recognition of the cognitive nature of complexity fits well with the use of the word "complex" in common parlance" [23] Some of the problem of trying to represent people's complex thoughts about SES is that the actors have cognition above that of simple programmable characteristics. Thus, we start "from observing ... the way decision-makers represent problems in their mind" [23] These representations need to be simplified. One way to do this is to only model the behaviors of fishers that change the environment, such as gear and boat choices. We did discuss how their choices might be influenced by their social norms but inclusion of suitable models of these cognitive processes was not a priority for us.

The ABM was, thus, to represent, in some detail, a small part of the whole system which contains multiple ecosystems; multiple types of stakeholders; multiple natural resources; and multiple socio-cultural connections. A first step in model development was to abstract the realworld system to the key ecosystems and livelihoods of interest for the study. In the case of Kenya, we concentrated on the artisanal fishers and the shallow coastal water environment which contains coral reef in addition to the inshore farmlands as these are two of the most important resources for poor people. 


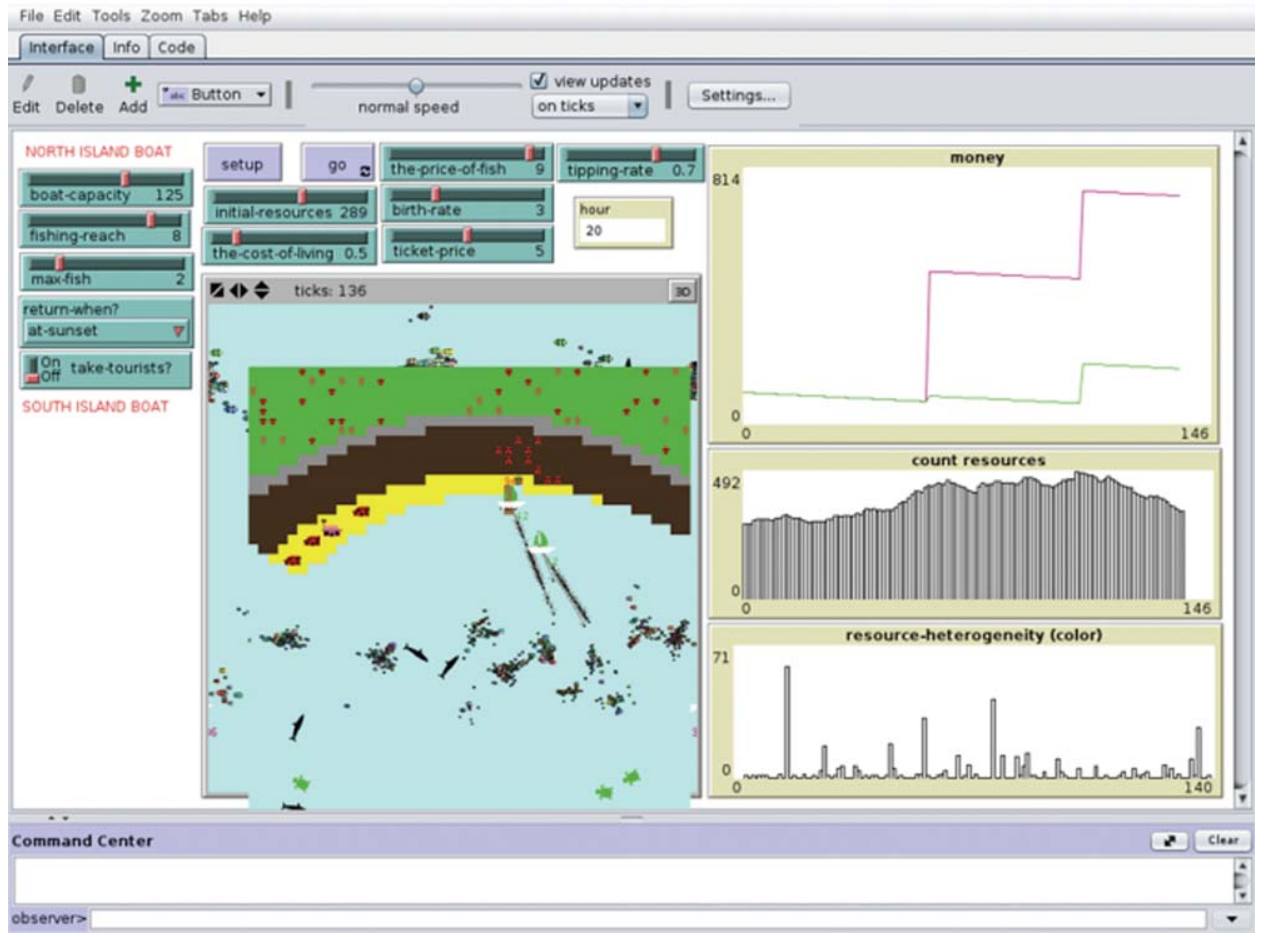

A screen shot of a model for fisheries.

As noted in the section Background, Including the Setting of the Problem, we based our data gathering methodology on the companion modeling approach. We adapted it to understand how actors access different types of resources; how this influences their decision making; what are the important decision points now and in the past; and what are the power relations that shape control over access to and use of coastal resources. We tailored additional methods such as social network mapping (see http://netmap.wordpress.com/about/) to elicit information about actors as observed by the participants themselves. Rather than collecting detailed qualitative information, the activities allows different homogeneous "focus groups" of stakeholders to broadly characterize their networks, the types of interaction they perceive, and the actors they consider to be influential.

Our approach, using "structured subjectivity" data gathering methods (see Introduction) produced data in a format more readily utilized within the ABM. Thus, it was possible to generate agent-based modeled scenarios based on actors' own hypotheses. Further, at a workshop held in Ukunda, on the south Kenya coast-and using the University of Oxford's Behaviour Composer (see http://resources. modelling4all.org/Home/behaviour-composer-direct-to-ne tlogo-guide)-BMU members and other representatives of coastal communities were able to run the model to explore impacts of different gear usage on the fish stock, generate a number of possible scenarios, articulate feedback loops, and came up with discussion points that could be incorporated into the model to make it better suited for use in real situations.

Thus, the agents (actors) within our ABM are informed by real actors in the field and, as a result, have characteristics that reflect actors' reality and are more recognizable to end users. The scenarios generated by our ABM (using NetLogo) typically look like Figure 2.

The model is a dynamic process and end users can, and have, engaged with the model, running brief simulations using different inputs to test scenarios generated. To date, there is no detailed publication available of our ABMs but these are under construction. The workshop (mentioned) is reported at http://weadapt.org/knowledgebase/adaptation-decision-making/wd-nace-scenarioskenya and describes the interaction with domain experts. At the workshop, we first explained the main ideas captured in the model, and then, demonstrated how the software and simulation works (using laptops).

Links to model codes are available: NetLogo model codes are at: http://modelingcommons.org/browse/one_model/ 3435; Modelling4all model codes: http://m.modelling4all. 


\section{FIGURE 3}

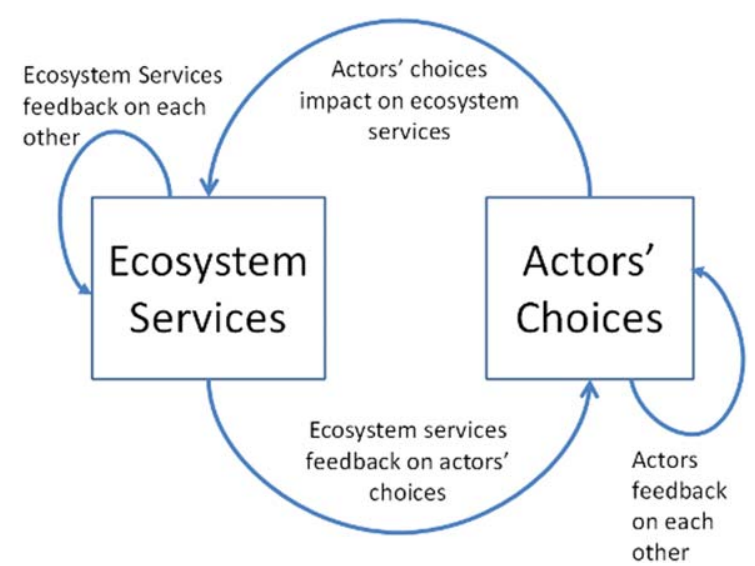

The original project "metamodel."

org/m/?frozen=tB3AfKKQQMU_z2Uxm2E14f \&MforAllMod $\mathrm{el}=1$. At a deeper level, ABMs allow people to think "scientifically" without the mathematics. The highly visual NetLogo interface allows people to "do the maths" by manipulating symbols moving through space time. They know their real world and, because the interface looks (something) like their world, they can spot any deviation from [their perception of] "reality" within the model.

\section{ON EXPERIENCE OF "TOP-DOWN" MODELING (USING UML)}

Our conceptual model (CoSMoS "domain model”) can be considered similar to the "archetypical cognitive maps [that] usually are approximate and simplified versions of scientific insights-and are called metamodels, "stylized facts," or simply correlations" [8] and, like our metamodels (cf. initial project "metamodel" and its corresponding UML diagram, Figures 3 and 4), it is probably best to assume that they are incomplete and imperfect. Notwithstanding, the combining "of value orientations and cognitive maps which make up worldviews ... provides the basis for the construction of ... model-based narratives" which can be used to "support strategic decision-making, as well as heuristic exploration in complex sustainability related macro-problems" [8].

Thus, our UML model is of the whole system-or as close to the whole system as we can get from the actors we have involved (often overlapping with the same actors who informed the ABM). We did not need to use UML but using a formalized, structured language we constrained individual's conceptual maps so that they were comparable and collatable across actors. The first task was to achieve a sufficient understanding of the way the locally based researchers and experts perceive the discrete "classes" and linkages in their minds. This allowed a rationalized understanding of the system which could be compared with what the day-to-day decision taker knows. We must accept that the framing of our questions

\section{FIGURE 4}

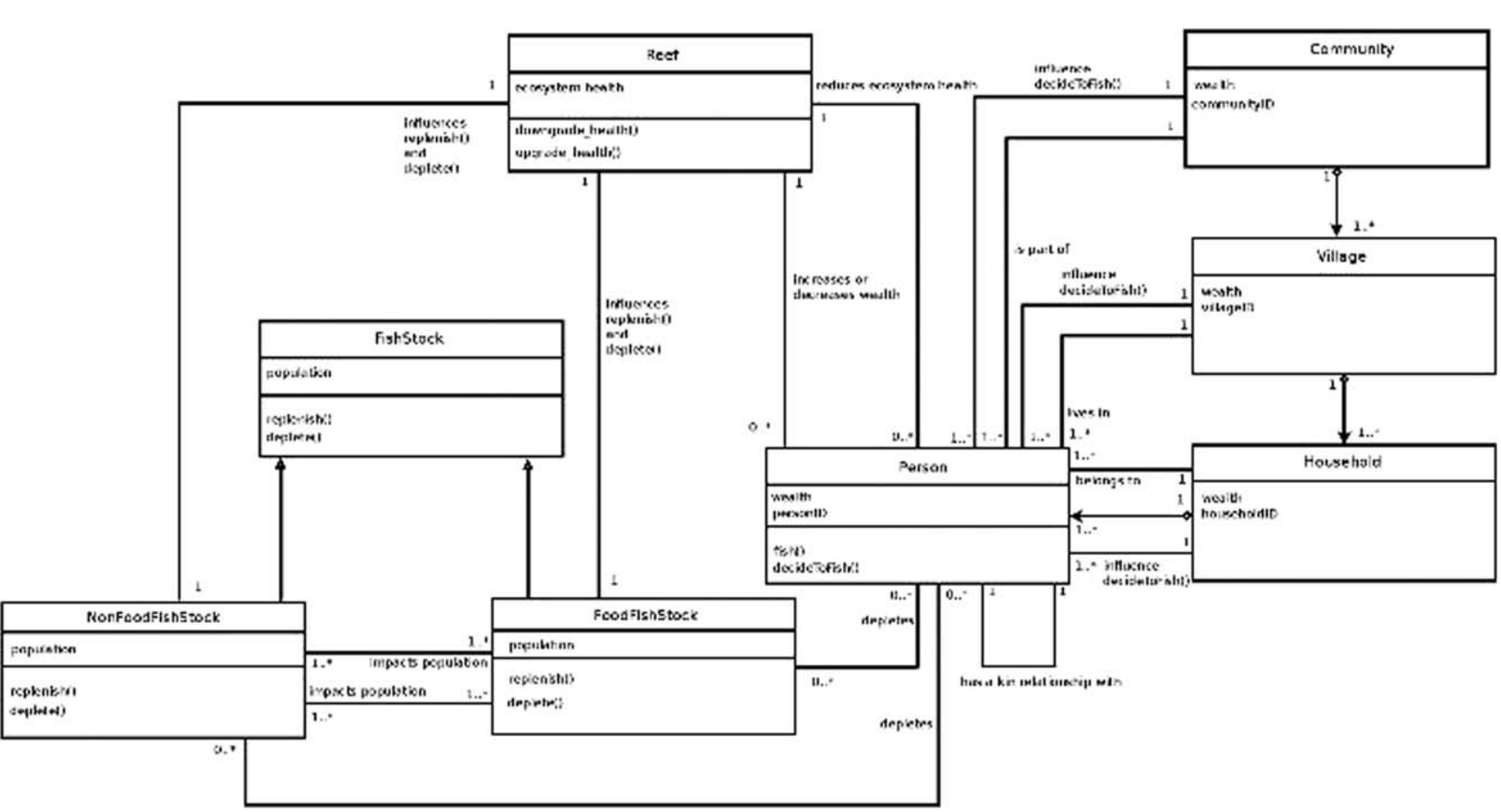

The computer systems unpacking of the initial "meta model" aka the UML Class Diagram. 


\section{FIGURE 5}

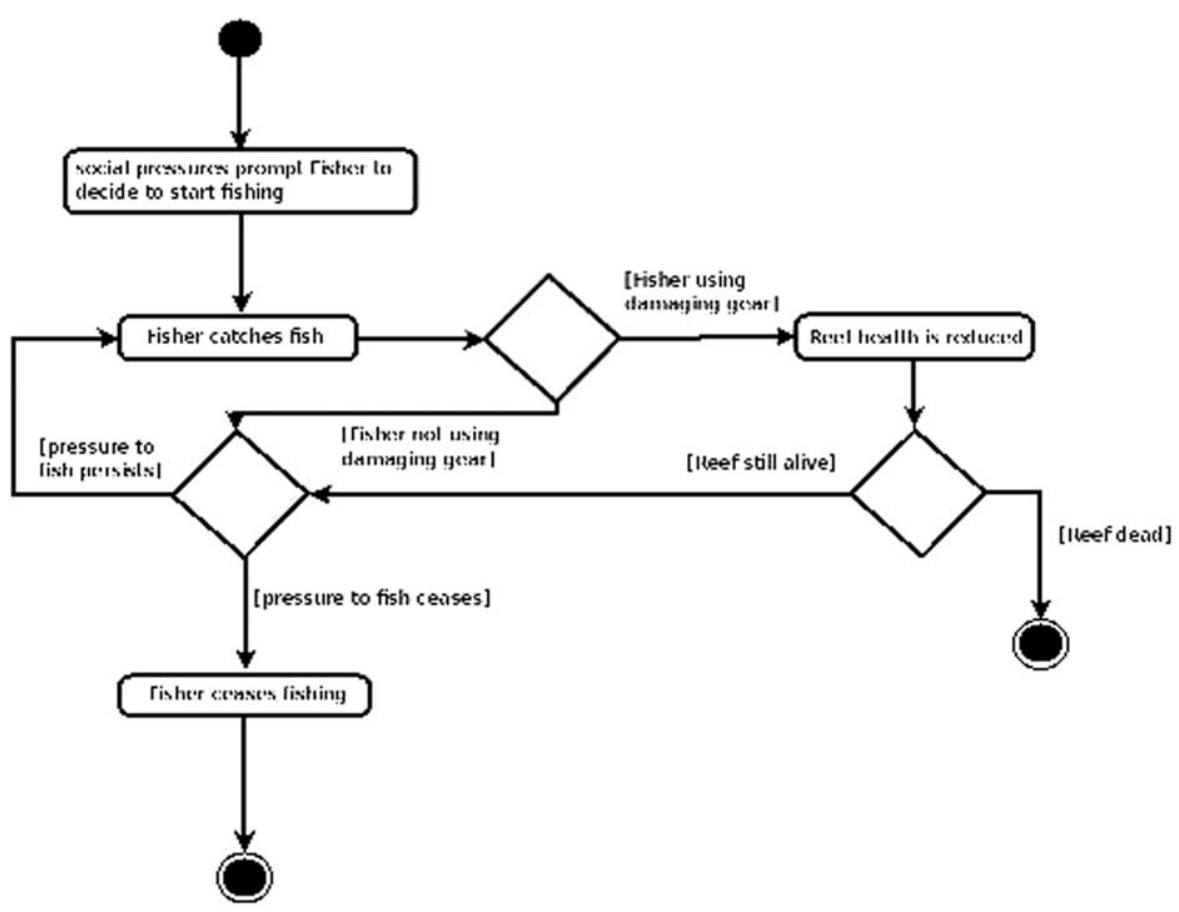

Activity diagram for fishing.

influenced what our informants told us, but this reinforces our perceived need for structured subjectivity rather than using methods which allow subjects to determine their own framework.

The UML descriptions are models of how people think the system is: they are the structured subjective views of the domain experts (in our case, the three primary domain experts were a Kenyan social scientist and ecologist who were intimately knowledgeable about the subject matter and a political economist). The role of the computer scientist was to ask questions of these domain experts, questions which the domain experts often innately knew but which needed to be made explicit in the model, such as "why does the fisher fish?" By accessing their understandings, we can access a window onto their cognitive (or mental) maps of how the world works. Once made explicit (in the diagram) this conceptual map can then be tested more rigorously (i.e., validated) with the actual social actors involved. The UML diagrams (a single class diagram, see Figure 4; a series of state diagrams for each of the classes of concern, not reproduced here; and a series of activity diagrams for important processes-see Figure 5 for an example) were, thus, iteratively coproduced.

The purpose of the UML diagram set is twofold: first, it provides a formal but real-world-based notation of the system which is a useful heuristic device. It has proved useful to communicate the system to stakeholders from different disciplinary backgrounds, but it is also useful to describe the system, and its processes, to modelers who are not themselves domain experts. It does so in a notation which, thus, introduces a structure which would otherwise be lacking in these descriptions. Second, it starts to provide a framework within which we may start to think about how to link different models and models of different parts of the system and to do so in a structured, systemic way rather than using ad-hoc soft linkages.

The output of this part of our concept-testing project, $\mathrm{NB}$ we set out only to take the first step in the CoSMoS process (Figure 1) and test the domain modeling approach with a SES, are the series of UML diagrams. The modeler was initially given a schematic or cartoon model which had been prepared at the outset of the project and asked "to populate it"-that is to unpack the twin boxes of "ecosystem services" and "actors choices" and relevant interlinkages and feedbacks shown in Figure 3.

Several iterations of work by the computer programmer/ modeler, in communication with various domain experts, resulted in an expansion and a formalization of Figure 3 to become a UML class diagram as shown in Figure 4.

This class diagram is supplemented by a series of state diagrams for each class. These are not reproduced here. Examples of multiple UML diagrams including detailed 
state diagrams can be found in Greaves et al. [21]. The class diagram is also complemented by an activity diagram for significant activities that have an impact on the ecosystem. One of these activity diagrams is reproduced here.

We have now "truth grounded" these diagrams and what they represent with the stakeholders who have informed the research through which they were created, and generally they hold valid. However, they have also been a useful heuristic tool within the project itself to allow cross-disciplinary communication both of deeper cross-disciplinary understanding of the bigger system but also communication of purpose. " $[\mathrm{M}]$ odeling ecological or environmental problems is a challenge when humans are involved" and any unifying modeling approach must bring together the knowledge of many different experts from different disciplines, to be able to compare their perceptions [24]. We believe that our UML diagram set starts to do this.

\section{DISCUSSION}

Our initial plan within the project was to use a single $\mathrm{ABM}$ of the system as "an alternative approach that models social life as interactions among adaptive agents who influence each other in response to the influence they receive" [25]. We successfully recorded relevant primary data from our case study areas; we produced (social) network maps and associated data; and then started to produce an ABM based on these social data and also physical data about the natural ecosystem. Our model, based on actors' different understandings of the system, of course require a "qualitative validation" to provide a "reality check" [24] with the actors who have informed our model and other significant stakeholders within the system.

In the process of qualitative validation/reality checking, we quickly recognized the problem of "bolting together" data and inputs from different epistemic backgrounds within a single ABM. We, therefore, modified our plan, trying to understand a framework within which we could conceive of coupling or "nesting" separate, localized ABM within some "meta" framework. By separating out two modeling processes, while at the simulation stage there is still some need to couple social and biophysical aspects, we found that there is no formal requirement to couple the $\mathrm{ABM}$ and the framework (provided in UML). They operate independently at different scales, describing the same social-ecological reality but in different ways. Thus, rather than attempting to produce one all encompassing, highly complex simulation model as might be suggested by Figure 1 , we opted to create the separate models of the whole system's framework using UML within the CoSMoS approach, while using ABM (NetLogo founded in the Modelling4All philosophy) to simulate local-level "realities."

ABMs prove excellent at the local level but the approach did not scale up particularly well. Thus, we explored the use of UML diagrams to describe this whole system and this allows us to start to think about how to link up to higher scales of governance. We were persuaded by Özemi and Özemi that "Formal validation of these cognitive maps is not possible because the maps operate on different understandings of the system" [24]. Thus, we accepted that the model created in UML is really such a cognitive map-or rather an aggregation of several actors' cognitive maps. This means that what we are modeling in UML is really rather different from the ABM. We also recognize that our conceptual model (CoSMoS "domain model") is more of an exploratory tool rather than a formal "reproduction" of the SES (as might be suggested in Figure 1). However, as the UML diagram set represents the influences which actors receive both from other actors within the system and from other (ecological) elements within the system, it is, therefore, directly testable against what people tell us, and also indirectly testable against observed data. Thus, while we do not need to validate the UML social-ecological model in the same way as the ABM, we can cross-validate between the $\mathrm{ABM}$ and the UML. Social simulation also introduces the idea of "cross-validation" [3] with both a qualitative and a quantitative component to comparison at different scales.

By bringing structured-subjective data written into UML together with similar data coded into an ABM it become possible to generate agent-based modeled scenarios based on actors' own hypotheses and validate/critique the simulations the ABMs produce separately from the conceptual model written in UML. Naturally, the formal modeling process (assumptions, coding, etc.) all still needs to be checked so that the modeler has confidence in the model, but for SES social science application we believe that we can be content with generating useful and potentially truthful scenarios. Because our modeling processes at both levels have been conceptually "truth grounded" with a relatively disparate group of stakeholders, we have a reasonably high level of confidence in the whole modeling approach. End user and stakeholders have engaged with the embryonic ABM, running brief simulations using different inputs to test scenarios generated and they have also qualitatively agreed with the cartoon model (unimplemented in code) as presented by in UML. Thus and here is an argument for multiple models, validation can come in the comparison between very different models (e.g., UML and ABM) and empirically between any one of those models (in our case the ABM) and observed "reality."

We also needed a clear framework within which we can understand how models of different parts of the system can be interlinked. But the overarching model written in UML is, we would argue, not a simulation of the system or a half-way-house toward a simulation but rather a map of how the system is conceived. It is a conceptual model. This model models how people think about the system: thus, it must contain the detailed information (and all the 
classes) that people use to conceive of the system. It needs a KIDS approach. If we wanted to simulate this conceptual model (CoSMoS domain model: a stage we had never planned within the project) the modeler might well naturally apply a KISS approach, but this would need to be carried out iteratively with the domain experts so as to maintain cross-disciplinary trust.

This multiple model approach also allows us to deal with emergent properties in a novel way. For example, power relationships between actors obviously influence how actors behave: thus, such relationships are built in to an $\mathrm{ABM}$ as actor characteristics. But power relations can also be emergent properties of how actors have exploited resources. In the UML representation then, as it is a model of the whole system, it does not have power relationships built in because here they are an emergent property of the social relationships (i.e., the right-hand feedback loop in Figure 3). This makes our ABM apparently more detailed and makes it, by comparison, look more like the type of ABM we started with, the natural science simulation model. But it also emphases that the UML and the ABM are different ways of representing the same reality: while there is not such a big difference notable between the UML and the ABM in our case (we were not explicitly modeling heterogeneity), they can be used in very different ways.

Finally, our experience is that modelers need to quickly learn when to use simulation models and when to use conceptual models as alternative ways to communicate, and social scientists need to understand the protocol and methods by which modelers can simulate.

\section{CONCLUSIONS}

There is no current methodology agreed on for how to represent such SES. Complex simulation modeling is required to provide decision takers and policy advisors with multiscale, multisector models to support decisions with respect to poverty alleviation, and ecosystem services decisions: but this modeling should be as simple as possible for the task in hand and readily interpretable to users. Engaging model end users in cocreation is one way to achieve this.

The conceptual model provided by the UML process provides a general landscape within which the structurally realistic model $(\mathrm{ABM})$ can exist and be understood. This approach, we argue, helps us develop tools to capture and conceptualize whole ecosystem dynamics and processes. We further argue that it can be used successfully when there is a need to understand the system from both topdown and bottom-up: that is as "a system" and also as the constituent stakeholders understand it.

\section{ACKNOWLEDGMENTS}

With acknowledgments for domain knowledge input particularly from David Obura, CORDIO, Kenya; Emmanuel Yaa, Mombasa, Kenya; and Nilufar Matin, SEI.

\section{REFERENCES}

1. Ackhoff, R. Redesigning the Future; Wiley: New York, 1974.

2. Law, J. After Method: Mess in Social Science Research; Routledge: London and New York, 2004.

3. Edmonds, B.; Moss, S. From KISS to KIDS an 'anti-simplistic modelling approach'. In: Multi Agent Based Simulation; Moss, S.; Davidsson, P., Eds.; Springer: Berlin, 2005; pp 130-144.

4. Eden, S.; Donaldson, A.; Walker, G. Structuring subjectivities? Using Q methodology in human geography. Area 37, 2005, 413-422.

5. Bharwani, S. Understanding complex behavior and decision making using ethnographic knowledge elicitation tools (KnETs). Soc Sci Comput Rev 2006, 24, 78-105.

6. Rahman, A.; Mallick, D.; Haque, N.; Nishat, A. Trends in Natural Resource Management in Bangladesh: Looking for Integration and a New Institutional Framework; Bangladesh Centre for Advanced Studies, UK Department for International Development, Natural Resource Management Workshop, Paper Series \# 2, Dhaka, 2002.

7. Coulthard, S.; Johnson, D.; McGregor, A. Poverty, sustainability and human wellbeing: A social wellbeing approach to the global fisheries crisis. Global Environ Change 2011, 21, 453-463.

8. de Vries, B.; Petersen, A. Conceptualizing sustainable development: an assessment methodology connecting values, knowledge, worldviews and scenarios. Ecol Econ 2009, 68, 1006-1019.

9. Anselme, B.; Bousquet, F; Lyetc, A.; Étienne, M.; Fady, B.; Le Page, C. Modelling of spatial dynamics and biodiversity conservation on Lure mountain (France). Environ Modell Software 2010, 25, 1385-1398.

10. Bousquet, F; Barreteau, O.; Le Page, C.; Mullon, C.; Weber, J. An environmental modelling approach. The use of multiagents simulations. In: Advances in Environmental and Ecological Modelling; Blasco, F; Weill, A., Eds.; Elsevier: Paris, 1999; pp 113-122.

11. Bousquet, F.; Le Page, C. Multi-agent simulations and ecosystem management: A review. Ecol Modell 2004, 176, 313-332.

12. Étienne, M. Companion modelling: A tool for dialogue and concertation in biosphere reserves. In: Biodiversity and Stakeholders: Concertation Itineraries. Biosphere Reserves-Technical Notes 1; Bouamrane, M., Ed.; UNESCO: Paris, 2006; pp 4453.

13. Étienne, M.; Cibien, C.; Génot, J.-C. An example of companion approach which could be used during the periodic review. In: Dialogue in Biosphere Reserves. References, Practices and Experiences. Biosphere Reserves-Technical Notes 2; Bouamrane, M., Ed.; UNESCO: Paris, 2007; pp 53-59. 
14. Étienne, M.; Du Toit, S.; Pollard, S. ARDI: A co-construction method for participatory modelling in natural resources management. Ecol Soc 16(1), Article 44, 2011.

15. Kemp-Benedict, E.; Bharwani, S.; Fischer, M. Using matching methods to link social and physical analyses for sustainability planning. Ecol Soc 15(3), Article 4, 2010.

16. Andrews, P.; Polack, F.; Sampson, A.; Stepney, S.; Timmis, J. The CosMoS Process: A Process for the Modelling and Simulation of Complex Systems; University of York: York, 2010.

17. Polack, F.; Andrews, P.; Ghetiu, T.; Read, M.; Stepney, S.; Timmis, J.; Sampson, A. Reflections on the simulation of complex systems for science. In: ICECCS: Fifteenth IEEE International Conference on Engineering of Complex Computer Systems; IEEE Press, 2010; pp 276-285.

18. Larman, C. Applying UML and Patterns: An Introduction to Object-Orientated Analysis and Design and Iterative Development; Prentice Hall: Westford, Mass., 2005.

19. Booch, G.; Rumbaugh, J.; Jacobson, I. The Unified Modelling Language User Guide; Addison-Wesley Longman: Reading, MA, 2005.

20. Fowler, M. UML Distilled: A Brief Guide to the Object Modelling Language; Addison-Wesley: Boston, 2004.

21. Greaves, R.; Polack, F; Forrester, J. CoSMoS in the context of social-ecological systems research. In: Proceedings of the 2012 Workshop on Complex Systems Modelling and Simulation: CoSMoS; Stepney, S.; Andrews, P.; Read, M., Eds.; Luniver Press: Frome, UK, 2012; pp 47-76.

22. Mashanova, A.; Law, R. Resource dynamics, social interactions, and the tragedy of the commons. In: Micro, Meso, Macro: Addressing Complex Systems Couplings; Liljenström, H., Svedin, U., Eds; World Scientific Publishing, Singapore, 2005; pp 135-146.

23. Fioretti, G.; Visser, B. A cognitive interpretation of organisational complexity. Emergence: Complexity and Organisation (E:CO) 2004, 6, 11-23.

24. Özemi, U.; Özemi, S. Ecological modelling based on people's knowledge: A multi-step fuzzy cognitive mapping approach. Ecol Modell 2004, 176, 43-64.

25. Macy, M.; Willer, R. From Factors to Actors: computational sociology and agent-based modelling. Annu Rev Soc 2002, 28, 143-166. 